http://jmscr.igmpublication.org/home/ ISSN (e)-2347-176x ISSN (p) 2455-0450

crossref DOI: https://dx.doi.org/10.18535/jmscr/v9i1.45

\title{
Risk Factors of Gynaecological Cancers-A Hospital Based Case Control Study
}

\author{
Authors \\ Dr Harini. $T^{1}$, Dr Priyadarsini. $S^{2}$ \\ ${ }^{1}$ Junior Resident, Dept. of Obstetrics \& Gynaecology, TDMCH, Alappuzha \\ ${ }^{2}$ Asst. Professor, Dept. of Obstetrics \& Gynaecology, GMC, Trivandrum
}

\begin{abstract}
Objective: The aim of this study was to determine the risk factors associated with various gynaecological malignancies.

Materials and Methods: All women's attending gynaecological OPD and radiotherapy OPD who were diagnosed to have malignancy of the cervix, endometrium, ovary and other malignancy of the genital tract are taken as cases and those women without malignancy as excluded by pap smear, ultrasound examination and endometrial sampling are taken as controls. These population are studied for one year period from Jan 2016 to Jan 2017.

Results: Among the risk factors studied presence of diabetes increases the risk of ovarian and endometrial malignancy. The odds ratio for carcinoma ovary and carcinoma of the endometrium were 3.1 and 4.4 which is statistically significant. Similarly patients with hypertension and obesity were at increased risk of carcinoma of the endometrium. The odds ratio were 3.2 and 3.5 respectively. This cause the shift in the trend of gynaecological malignancy more towards ovarian and endometrium.

Conclusion: Ovarian and endometrial cancer is on the rise compared to cervical cancer. Also an association has been found between diabetes, hypertension, obesity and malignancy. These observations suggest that the possibly environmental and lor life-style factors affecting the change had influence on all the age groups.
\end{abstract}

Keywords: Gynaecological cancer; Obesity, Hypertension; risk factors.

\section{Introduction}

Gynaecological cancers form a huge burden of morbidity and mortality around the world. $70 \%$ of women present at an advanced stage of the disease, which results in poor survival and high mortality. Identification of risk factors can help in early screening and diagnosis of these patients thereby decreasing the morbidity and mortality.

Endometrial cancer is the most common gynaecologic malignancy in developed countries. The association of endometrial cancer with age, BMI, race, family history, polycystic ovary, diet, physical activity, smoking, parity, breast feeding, socio economic status etc are known. Type 2 endometrial cancer is associated with excess age, non white race and obesity. Women with infertility are reported to have an elevated risk of endometrial neoplasm.

Cervical cancer is the second most common cancer in the world. Around $80 \%$ cases are detected in developed countries. It has been found out that HPV infection is the most powerful epidemic factor associated with cervical cancer. Highest incidence is seen in the 45 - 60 year age 
group. A study conducted at the WHO collaborative center at Uppsala University determined that tobacco smoking, alcohol consumption and the use of narcotics were risk factors but not causative agents. The incidence of bacterial vaginosisis seen to be higher in patients with pre-cancerous lesions of cervix.

Ovarian cancer is one of the most common gynaecological cancers that ranks third after cervical and uterine cancer. It has the worst prognosis as it is usually asymptomatic and due to lack of proper screening tests. Various lifestyle, environmental and genetic factors increases the risk of ovarian cancer. Many factors such as pregnancy, lactation, and oral contraceptive pills play a role in reducing the risk of ovarian cancer.

\section{Objective}

To study the risk factors of gynaecological malignancies like carcinoma endometrium, carcinoma cervix and carcinoma ovary in our Hospital.

\section{Methodology}

This is a Hospital based case control study. All women attending gynaecology OPD and radiotherapy OPD in Govt. TD medical college Alappuzha during a one year perod was included in the study. Assuming a prevalence of $20 \%$ for one of the major risk factors like obesity in the control group, with an Odds ratio of $3, \alpha$ of 0.05 and power of $80 \%$, ratio of cases to control 1 , the sample size is estimated as 65 cases and 65 controls. Those women who are diagnosed to have malignancy of cervix, endometrium, ovary and any other genital tract malignancies are taken as cases. They are age matched women without malignancy as excluded by pap smear, ultrasound examination and endometrial sampling are taken as controls.

\section{Results}

A total of 136 women were studied .Out of which 68 women with malignancy are taken as cases and 68 women are taken as controls.

Table 1: Association of risk factors with cancer cervix

Sl. No. Parameters Cancer cervix control $\begin{aligned} & \text { Odds Ratio } \\ & 95 \% \text { CI }\end{aligned}$ p value\#

\begin{tabular}{|c|c|c|c|c|}
\hline Age group & & & & \\
\hline$<50$ & $1(2.4)$ & 41 (97.6) & 1 & $<0.001 *$ \\
\hline$\geq 50$ & $15(35.7)$ & $27(64.3)$ & $22.7(2.8-182)$ & \\
\hline
\end{tabular}

2 Menstrual history

$\begin{array}{lllcc}\text { Premenopausal } & 1(2.4) & 41(97.6) & 1 & <0.001^{*} \\ \text { Postmenopausal } & 15(35.7) & 27(64.3) & 22.7(2.8-182) & \end{array}$

3 Parity

$\begin{array}{lclcc}\text { Nullipara } & 2(50) & 2(50) & 4.7(0.6-36) & 0.16 \\ \text { Multiparous } & 14(17.5) & 66(82.5) & 1 & \end{array}$

$4 \quad$ Diabetes

$\begin{array}{lllcl}\text { Present } & 9(27.3) & 24(72.7) & 2.4(0.7-7.1) & 0.12 \\ \text { Absent } & 7(13.7) & 44(86.3) & 1 & \end{array}$

5 Hypertension

$\begin{array}{lllcc}\text { Present } & 7(21.2) & 26(78.8) & 1.3(0.4-3.7) & 0.68 \\ \text { Absent } & 9(17.6) & 42(82.4) & 1 & \end{array}$

6 Obesity

$\begin{array}{lclcc}\text { Normal } & 6(14) & 37(86) & 1 & 0.22 \\ \text { Obese } & 10(24.4) & 31(75.6) & 1.9(0.6-6) & \end{array}$


Association between risk factors and cervical cancer was assessed using chi square test and the strength of association was estimated using odds ratio. It was found that age group more than or equal to 50 years, postmenopausal women, high parity were at risk of developing cervical cancer. People with age group more than or equal to 50

Table 2: Association of risk factors with cancer ovary

SI. No. Parameters Cancer ovary control 1

Age group

$<50$

$\geq 50$

7 (14.6)

$20(42.6)$

$41(85.4)$

$27(57.4)$

Odds Ratio 95\% CI

2

Menstrual history

Premenopausal

Postmenopausal

$6(12.8)$

$21(43.8)$

$41(87.2)$

$27(56.3)$

1

$4.4(1.6-11.6)$

yrs are 22.7 times having significantly higher risk to develop cervical cancer compared to their age counterpart ( $\mathrm{p}$ value 0.001). Postmenopausal women have 22.7 times significantly higher chance to develop cervical cancer compared to premenopausal women ( $\mathrm{p}$ value 0.001 ).

1
$5.3(1.9-14.8)$

$0.001 *$

3

\section{Parity}

Nullipara

Multiparous

$\begin{array}{lll}1(33.3) & 2(66.7) & 1.3(0.11-14) \\ 26(28.3) & 66(71.7) & 1\end{array}$

0.84

4

Diabetes

Present

Absent

$17(41.5)$

$10(18.5)$

$24(58.5)$

$3.1(1.2-7.8)$

$0.01 *$

5 Hypertension

Present

$16(38.1)$

$44(81.5)$

Absent

$11(20.8)$

$26(61.9)$

$42(79.2)$

$2.3(0.9-5.8)$

0.06

6 Obesity

Normal

Obese

$11(22.9)$
$16(34)$

$37(77.1)$

$31(66)$

1

$1.7(0.7-4.2)$

0.22

Note:\# p value based on chi-square test, $*$ statistically significant $(\mathrm{p}<0.05)$, CI confidence interval

Association between risk factors and ovarian cancer was assessed using chi square test and the strength of association was estimated using odds ratio. It was found that age group more than or equal to 50 years, premenopausal status, nulliparous women and individual with diabetes were at risk of developing ovarian cancer. People with age group more than or equal to $50 \mathrm{yrs}$ are 4.4 times having significantly higher risk to develop ovarian cancer compared to their age counterpart ( $\mathrm{p}$ value 0.001). Postmenopausal women have 5.3 times significantly higher chance to develop ovarian cancer compared to premenopausal women ( $\mathrm{p}$ value 0.001). Nullipara have 1.3 times higher chance to develop carcinoma ovary but this was not statistically significant. Diabetes have 3.1 times significantly higher chance to develop ovarian cancer. 
Table 3: Association of risk factors with endometrial cancer

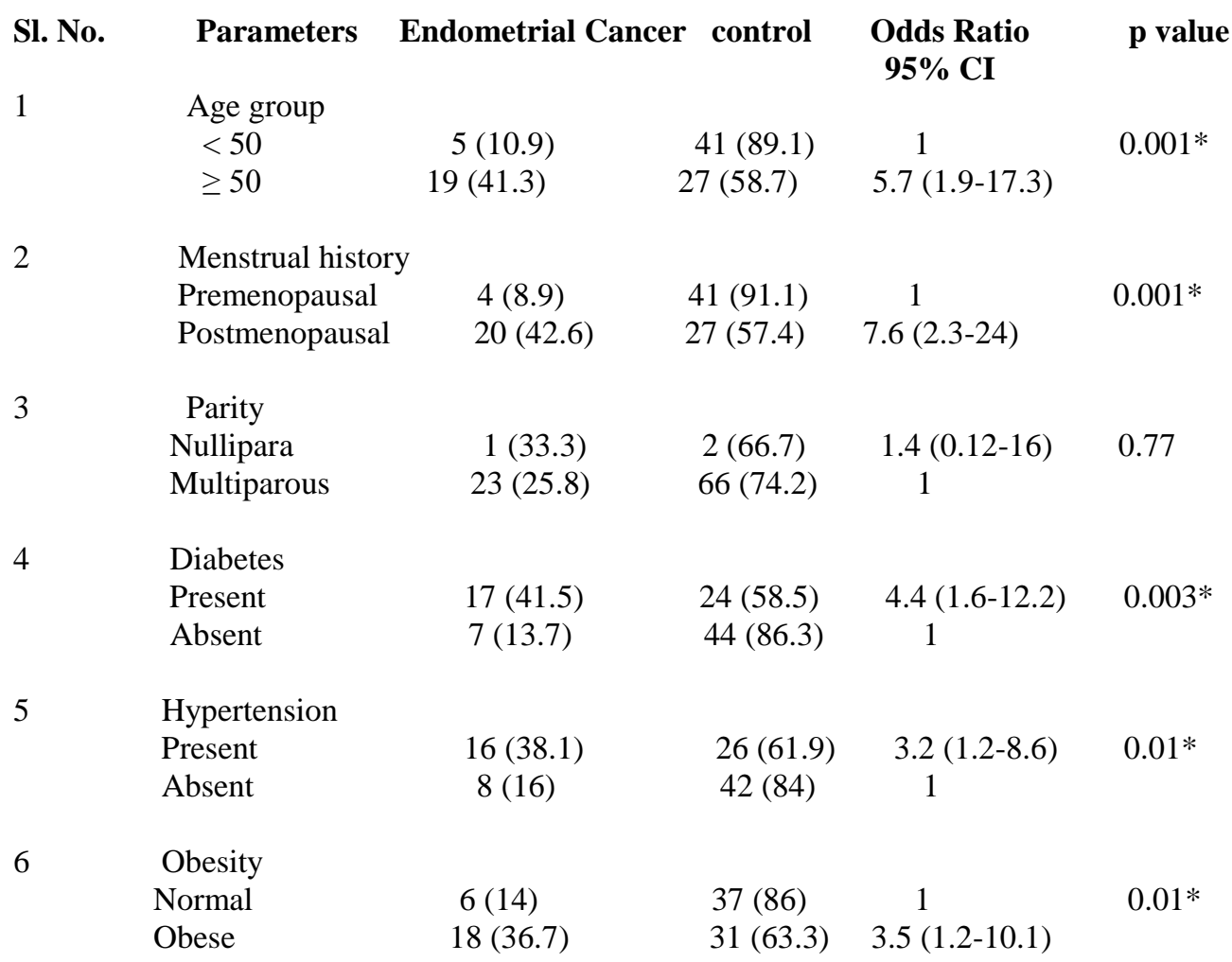

Note: \# p value based on chi-square test, * statistically significant $(\mathrm{p}<0.05)$, CI confidence interval

Association between risk factors and endometrial cancer was assessed using chi square test and the strength of association was estimated using odds ratio. It was found that age group more than or equal to 50 years, postmenopausal status, nulliparous women ,individuals with diabetes, hypertension and obesity were at risk of developing endometrial cancer. People with age group more than or equal to $50 \mathrm{yrs}$ are 5.7 times having significantly higher risk to develop endometrial cancer compared to their age counterpart ( $p$ value 0.001). Postmenopausal women have 7.6 times significantly higher chance to develop endometrial cancer compared to premenopausal women ( $\mathrm{p}$ value 0.001 ). Nullipara have 1.4 times higher chance to develop carcinoma endometrium but this was not statistically significant. Diabetes, hypertension and obese people have 4.4, 3.2 and 3.5 times significantly higher chance to develop endometrial cancer.

\section{Discussion}

Among the population studied it was found that in carcinoma ovary and carcinoma of the endometrium diabetes has been found to increase the risk. The odds ratio for carcinoma ovary and carcinoma of the endometrium were 3.1 and 4.4 which is statistically significant. A study conducted by Nandagudi Srinivasa Murthy et al showed that there is convincing evidence about association of diabetes with the cancer of corpus uteri Among the population studied it was found that in carcinoma of the endometrium hypertension has been found to increase the risk .The odds ratio is 3.2 which is statistically significant. A study conducted by Nandagudi Srinivasa Murthy et al showed that there is convincing evidence about association of hypertension with the cancer of corpus uteri.4-9 Among the population studied it was found that in carcinoma of the endometrium obesity has been found to increase the risk. The odds ratio is 3.5 which is statistically significant. A study conducted by Nandagudi Srinivasa Murthy et al showed that there is convincing evidence about 
association of obesity with the cancer of corpus uteri. 4-9

\section{Conclusions}

The present study was conducted to find out the risk factors of gynaecological malignancies like carcinoma endometrium, carcinoma cervix and carcinoma ovary. We hypothesized that carcinoma endometrium and ovary is on the rise due to increasing rate of obesity, infertility and life style changes.

The findings are

- The common age of malignancy is around 45 to $50 \mathrm{yrs}$.

- Women with high parity are at increased risk for carcinoma cervix.

- It was found that ovarian malignancy constitutes about $39.8 \%$ followed by uterine and cervical cancer about $35.2 \%$ \& $23.6 \%$ respectively which shows there is a change in trend in gynaecological malignancy.

- Among the risk factors studied presence of diabetes, hypertension and obesity increases the risk of ovarian and endometrial malignancy which is statistically significant. This cause the shift in the trend of gynaecological malignancy towards ovarian and endometrium compared to previous data which shows cervical cancer.

\section{References}

1. Cancer Incidence and Patterns in Urban Maharastra-2001, Report to the State of Maharashtra on Status of Cancer, Indian Cancer Society, Cancer Registry Division Division, Mumbai, India. 2007(2)

2. Nandakumar A, Anatha N, Dhar M, et al (1995). A case-control investigation on cancer of the ovary in Bangalore, India. Int J Cancer, 63, 361-5. (2)

3. Miller BA, Ries LAG, Hankey BF, Kosary CL, Harras A, Devesa SS, Edwards BK (eds): SEER Cancer Statistics Review:
1973-1990. Bethesda, MD: National Cancer Institute; NIH publication 93-2789, 1993.Platz CE, Benda JA: Female genital tract cancer. Cancer 75:27\&294,1995

4. Pettersson B, Adami H, Lindgren A, Hesselius I: Endometrial polyps and hyperplasia as risk factors for endometrial carcinoma. Acta Obstet Gynecol Scand 64:65349, 1985

5. Kjaerbye-Thygesen A, Huusom LD, Frederiksen K, Kjaer SK (2005). Trends in the incidence and mortality of ovarian cancer in Denmark 1978-2002. Camparison with other Nordic countries. Acta Obstet Gynaecol Scand, 84, 1006-12.

6. La Vecchia C (2001). Epidemiology of ovarian cancer: a summary review. Eur J Cancer Prev, 10, 125-9. Muir CS, Waterhouse JAH, Mack T (1987). Cancer incidence in Five Continents, Vol. V, International Agency for Research on Cancer, Lyon, IARC Scientific Publication No. 88. 